\title{
Highly efficient interfacing of guided plasmons and photons in nanowires
}

\author{
Xue-Wen Chen, Vahid Sandoghdar, Mario Agio \\ Laboratory of Physical Chemistry and OptETH, \\ ETH Zurich, 8093 Zurich, Switzerland
}

\begin{abstract}
Successful exploitations of strongly confined surface plasmon-polaritons critically rely on their efficient and rapid conversion to lossless channels. We demonstrate a simple, robust, and broadband butt-coupling technique for connecting a metallic nanowire and a dielectric nanofiber. Conversion efficiencies above $95 \%$ in the visible and close to $100 \%$ in the near infrared can be achieved with realistic parameters. Moreover, by combining butt-coupling with nanofocusing, we propose a broad-band high-throughput near-field optical microscope.
\end{abstract}


Confined surface plasmon-polaritons (SPPs) have enormous potential for manipulating electromagnetic fields at optical frequencies and nanoscopic length scales ${ }^{1}$, with applications for interconnects ${ }^{2}$, field-enhanced spectroscopy and microscopy ${ }^{3 / 4|5| 6}$, sensing ${ }^{7}$, and quantum optics 8 . However, absorption of energy at optical frequencies by real metals makes propagation of confined SPPs very lossy in comparison with the transmission of photons in dielectric guides 2100 . Therefore, practical device proposals require a rapid and efficient conversion of SPPs into photons. In case of SPPs at planar interfaces, evanescent wave and grating couplers are very effective ${ }^{11}$. Thus, researchers have extended these schemes to guided modes of dielectric fibers $(\mathrm{DF})$ and metallic wires $(\mathrm{MW})$ in a side by side arrangement $\frac{812}{\text {, via }}$ tapers ${ }^{3 / 13[14 \mid 15}$, or by etching a grating on a MW nanocone ${ }^{16}$. However, efficient implementation of these approaches requires an interaction length greater than a wavelength $\frac{17}{}$, making losses an important issue. Furthermore, the critical dependence of evanescent coupling on the overlaps between the DF and MW modes, and the inherent wavelength dependence of grating coupling limit their bandwidths. In this Letter, we investigate the most straightforward and practical way of interfacing guided SPPs and photons between a MW and a nano $\mathrm{DF}^{18}$ in an axially-symmetric butt-coupling scheme. We investigate the conversion process of photons to SPPs as a function of wavelength and material, identifying the moulding of SPPs at the coupling interface as the condition for reaching efficiencies above $95 \%$ in the visible and close to $100 \%$ in the near-infrared range. Furthermore, we present a practically feasible scheme to overcome the long-standing conflict between strong field enhancement and high throughput in scanning near-field optical microscopy ${ }^{19}$, and to couple a quantum emitter to a propagating optical mode 20 .

We performed body-of-revolution finite-difference time-domain (FDTD) calculations ${ }^{21}$ to trace the propagation of the optical signal along the guides in a rigorous and computationally efficient manner, and to acquire an intuitive understanding of the mode conversion. 1(a) and (f) sketch the geometrical arrangements for interfacing SPPs on a silver ${ }^{\sqrt{22}}$ MW to guided photons in a silica DF and vice versa for a vacuum surrounding. The FDTD computational domain is restricted to the area enclosed by the dashed lines in 1(a) and (f), and its radial dimension is never less than two wavelengths. In each case, a steady-state SPP or a $\mathrm{TM}_{01}$ mode is launched from the left-hand side using the total-field/scatter-field technique ${ }^{21}$. The dashed red lines in 1(a) and (f) mark the source, which separates the scattered-field from the total-field region. The Drude model with conductivity is used to account for the dispersive 
properties of metals. The parameters are deduced by fitting the tabulated experimental $\operatorname{data}^{22 \mid 23}$ across a small spectral range $(100 \mathrm{~nm})$. 11(k) and (l) depict the time-averaged transverse magnetic field profiles of the radially-polarized modes at the vacuum wavelength of $\lambda=633 \mathrm{~nm}$ in the DF and MW with radii of $342 \mathrm{~nm}$ and $164 \mathrm{~nm}$, respectively. The snapshots of the magnetic field in 1(b)-(e) illustrate how SPPs travel on the surface of the MW and are converted to photons in the $\mathrm{TM}_{01}$ mode of the $\mathrm{DF}$. To determine the conversion efficiency $\eta$, we computed the ratio between the transmitted power in the guided mode right after the MW-DF interface and the incident power at the position right before it. The latter was calculated separately for an infinitely long MW or DF using the same source. In the example of 1 (a), the MW and DF yielded $\eta=95 \%$. We used a $2 \mathrm{~nm}$ grid here and found that the relative error between $1 \mathrm{~nm}$ and $2 \mathrm{~nm}$ grids is within $0.5 \%$. A grid size of $0.5 \mathrm{~nm}$ is used for 2(c) and 5. 1 $1(\mathrm{~g})-(\mathrm{j})$ show the reverse situation where light originates in the silica DF and is converted to SPPs of the silver MW with the same efficiency as in the previous case. The great advantage of the butt-coupling scheme is that the conversion between SPPs and guided photons takes place across an interface. This feature minimizes the impact of propagation losses, which significantly increase as wavelength and MW radius decrease $^{10}$. For instance, the MW at the wavelength considered in 1 would yeald losses as high as $0.72 \mathrm{~dB} / \mu \mathrm{m}$.

To explore the underlying coupling mechanism, in 2(a) we plot the time-averaged magnetic field for the region between the dashed lines in 1(b). We find that the SPP moulds around the end of the MW (see arrows in the inset of 2(c)) in such a way that the magnetic field assumes a maximum value at a certain distance from the axis on the MW-DF interface. Here, we tuned the radii of the two guides to obtain an SPP field profile that optimizes its coupling to the $\mathrm{TM}_{01}$ mode of the DF. 2(b) displays another example of the field distribution for larger MW and DF radii of $600 \mathrm{~nm}$ and $800 \mathrm{~nm}$, respectively. In this case the SPP magnetic field has three maxima, which clearly shows that the SPPs interfere and form a standing wave at the interface. Thus, a simple analysis based on mode-matching of the two guides $^{17}$ of 1 (a) only predicts $\eta=87 \%$ because it fails to account for the behaviour of SPPs. In fact, 2(c) shows that rounding off the MW edges facilitates this SPP folding process, improving $\eta$ and reducing reflections. Having demonstrated the possibility of very high conversion efficiency between SPPs and photons, it is important to examine the feasibility and reliability of this scheme for laboratory and technological applications. An important issue 
of concern is the fabrication tolerance. 2(d) displays $\eta$ as a function of the MW and DF radii at $\lambda=633 \mathrm{~nm}$, revealing that conversion efficiencies greater than $90 \%$ can be achieved even if variations of up to $15 \%$ take place for the radii. Moreover, we have verified that even a structure with an air gap of up to $50 \mathrm{~nm}$ between the MW and DF would yield a conversion efficiency greater than $94 \%$.

Next, we investigated the application of silver, gold ${ }^{22}$ or aluminium ${ }^{[23} \mathrm{MW}$ for operation at various wavelengths of interest in the visible, near infrared, and ultraviolet spectral regimes. We first found the radii by maximizing $\eta$ for a given wavelength using a mode-matching approach $\frac{17}{17}$ and then applied the FDTD technique to optimize them in a rigorous manner, by scanning the radii around the previously estimated values. 3(a) shows that for the case of silver MW, $\eta$ increases from $91 \%$ to $98 \%$, while reflection $R$ decreases from $2.1 \%$ to $0.4 \%$ as the wavelength grows over a spectral range greater than $500 \mathrm{~nm}$. 3(b) plots the values of the silver-MW and silica-DF radii corresponding to each optimized case. The results for the opposite propagation direction are very similar (not shown). 3(c) and (d) show that if silver is replaced by gold, $\eta, R$ and the corresponding radii yield similar values and trends. We note that around the telecommunication wavelength of $\lambda=1550 \mathrm{~nm}$, a gold MW transmits nearly $100 \%$ of the power to the DF. $3($ e) and (f) display the same analysis for an aluminium MW with emphasis on the ultraviolet region, where a rapid conversion of SPPs and photons becomes more critical due to the very short SPP propagation length. We find that even at a wavelength as short as $\lambda=266 \mathrm{~nm}, \eta$ can reach $89 \%$ if the DF and MW radii are respectively chosen to be $158 \mathrm{~nm}$ and $68 \mathrm{~nm}$, for which the SPP propagation loss in the MW amounts to $3.62 \mathrm{~dB} / \mu \mathrm{m}$.

Variation of the DF material can be used to engineer the coupling device to match the confined SPP modes on thin MWs. As inferred from 2 (a) and (b) the optimal radius of the MW can be reduced by a high-refractive-index DF also because the SPP standing wave on the coupling interface gets compressed by the larger wavenumber. 4(a) and (b) show the mode conversion properties for a gold MW at $\lambda=1550 \mathrm{~nm}$ as a function of the refractive index of the DF. The optimal radii of the MW and DF decrease and converge to nearly the same value for larger DF refractive indices, while in each case $\eta$ can be optimized beyond $99 \%$. As a last important property of concern, we examined the bandwidth of $\eta$ for a set of fixed MW and DF radii. 4(c) displays $\eta$ for a silver MW coupled to a silica DF in the visible range, and $4(\mathrm{~d})$ shows the same for a gold MW coupled to a silicon DF in the near-infrared 
regime. These results demonstrate that bandwidths greater than $150 \mathrm{~nm}$ are fully within reach for over $90 \%$ conversion of SPPs to photons and vice versa.

We now discuss an immediate consequence of efficient and broad-band SPP-photon conversion between a MW and a DF from both directions. Over the last decade, several reports have pointed out that SPPs can be focused tightly to nanoscopic regions at metallic

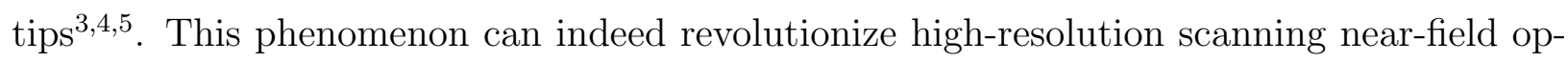
tical microscopy (SNOM), which has suffered from a very low transmission through small apertures 19 . However, so far there has been no viable approach for feeding optical energy into the SPP mode of the MW with a high efficiency. The device concept sketched in 5(a) provides an ideal solution for simultaneous intense and localized illumination as well as efficient collection. Furthermore, it is fully compatible with both state-of-the-art nanofabrication ${ }^{24}$ and scanning implementation of fluorescence, Raman, or other nonlinear nanoscopies $25 \mid 26$.

As a concrete example, we considered illumination at $\lambda=730 \mathrm{~nm}$ and fluorescence collection at $\lambda=780 \mathrm{~nm}$ from an emitter with a transition dipole moment oriented along the tip axis and embedded $5 \mathrm{~nm}$ below surface of a sample with a refractive index of 1.7 . The excitation light was launched from a DF of $410 \mathrm{~nm}$ radius, coupled to a gold nanocone with an initial radius of $200 \mathrm{~nm}$, and an opening angle of 14 degrees, which was terminated by a paraboloid. The tip was kept at a separation of $5 \mathrm{~nm}$ from the substrate. In 5(b) we plot the electric field intensity normalized to that of the source in the DF on a logarithmic scale for a zoom of a small region around the tip. The intensity is enhanced by more than 5 orders of magnitude and exhibits strong localization. 5(c) illustrates the emission process. We find that the radiated power is funnelled in the SPP and is then converted into the $\mathrm{TM}_{01}$ mode of the DF with an overall collection efficiency of about $70 \%$, which is only limited by propagation loss and radiation along the nanocone.

We have shown that confined SPPs of a MW can be converted into guided photons of a dielectric nanofiber with a very high efficiency and large bandwidth using a simple buttcoupling scheme. For a given wavelength between the visible and the near infrared spectral range, one can always obtain $\eta$ larger than $95 \%$ by an appropriate choice of radius and material of the MW and DF. We have found that moulding of SPPs at the MW-DF interface plays a fundamental role in achieving these performances. Furthermore, we have discussed an important application of butt-coupling in the context of SNOM and nanofocusing, proposing a scanning probe that combines broad-band and high-throughput with high-spatial resolu- 
tion. Efficient, broad-band and low-loss conversion of confined SPPs to guided photons is also key for a range of other applications such as high-resolution colour imaging27, on-chip manipulation and processing of quantum optical signals ${ }^{81920}$ for implementation of quantum networks $\frac{28}{}$, and actuation or sensing of physical, chemical, or biological processes at the molecular level, $[29$ paving the way for molecular-scale plasmonics.

We thank F. De Angelis and E. Di Fabrizio for fruitful discussions. This work was supported by ETH Zurich grant TH-49/06-1.

1 Barnes, W. L.; Dereux, A.; Ebbesen, T. W. Nature 2003, 424, 824-830

2 Conway, J.; Sahni, S.; Szkopek, T. Opt. Express 2007, 15, 4474-4484

3 Keilmann, F. J. Microscopy 1999, 194, 567-570

4 Babadjanyan, A. J.; Margaryan, N. L.; Nerkararyan, K. V. J. Appl. Phys. 2000, 87, 3785-3788

5 Stockman, M. I. Phys. Rev. Lett. 2004, 93, 137404

6 Yeo, B.-S.; Stadler, J.; Schmid, T.; Zenobi, R.; Zhang, W. Chem. Phys. Lett. 2009, 472, 1-13

7 Sharma, A.; Jha, R.; Gupta, B. IEEE Sens. J. 2007, 7, 1118-1129

8 Chang, D. E.; Sørensen, A. S.; Hemmer, P. R.; Lukin, M. D. Phys. Rev. Lett. 2006, 97, 053002

9 Chang, D. E.; Sørensen, A. S.; Demler, E. A.; Lukin, M. D. Nat. Phys. 2007, 3, 807-812

10 Novotny, L.; Hafner, C. Phys. Rev. E 1994, 50, 4094-4106

11 Raether, H. Surface Plasmons on Smooth and Rough Surfaces and on Gratings; Springer-Verlag, 1988

12 Lee, H. W.; Schmidt, M. A.; Tyagi, H. K.; Sempere, L. P.; Russell, P. S. J. Appl. Phys. Lett. 2008, 93, 111102

13 Bouhelier, A.; Renger, J.; Beversluis, M. R.; Novotny, L. J. Microscopy 2003, 210, 220-224

14 Janunts, N.; Baghdasaryan, K.; Nerkararyan, K.; Hecht, B. Opt. Commun. 2005, 253, 118-124

15 Ding, W.; Andrews, S. R.; Maier, S. A. Phys. Rev. A 2007, 75, 063822

16 Ropers, C.; Neacsu, C. C.; Elsaesser, T.; Albrecht, M.; Raschke, M. B.; Lienau, C. Nano Lett. 2007, 7, 2784-2788

17 Snyder, A.; Love, J. Optical Waveguide Theory; Springer, 1983

18 Tong, L.; Gattass, R. R.; B., A. J.; Sailing, H.; Jingyi, L.; Mengyan, S.; Iva, M.; Eric, M. Nature 2003, 426, 816-819 
19 Novotny, L.; Pohl, D. W.; Hecht, B. Opt. Lett. 1995, 20, 970-972

20 Domokos, P.; Horak, P.; Ritsch, H. Phys. Rev. A 2002, 65, 033832

21 Taflove, A.; Hagness, S. Computational Electrodynamics: The Finite-Difference Time-Domain Method, 3rd ed.; Artech House: Norwood, MA, 2005

22 CRC Handbook of Chemistry and Physics, 87th ed.; Lide, D., Ed.; CRC Press, 2006

23 Handbook of Optical Constants of Solids; Palik, E. D., Ghosh, G., Eds.; Academic Press, 1998

24 De Angelis, F.; Patrini, M.; Das, G.; Maksymov, I.; Galli, M.; Businaro, L.; Andreani, L. C.; Di Fabrizio, E. Nano Lett. 2008, 8, 2321-2327

25 Sánchez, E. J.; Novotny, L.; Xie, X. S. Phys. Rev. Lett. 1999, 82, 4014-4017

26 Ichimura, T.; Hayazawa, N.; Hashimoto, M.; Inouye, Y.; Kawata, S. Phys. Rev. Lett. 2004, 92, 220801

27 Kawata, S.; Ono, A.; Verma, P. Nat. Photon. 2008, 2, 438-442

28 Cirac, J. I.; Zoller, P.; Kimble, H. J.; Mabuchi, H. Phys. Rev. Lett. 1997, 78, 3221-3224

29 Zheng, Y. B.; Yang, Y.-W.; Jensen, L.; Fang, L.; Juluri, B. K.; Flood, A. H.; Weiss, P. S.; Stoddart, J. F.; Huang, T. J. Nano Lett. 2009, 9, 819-825 

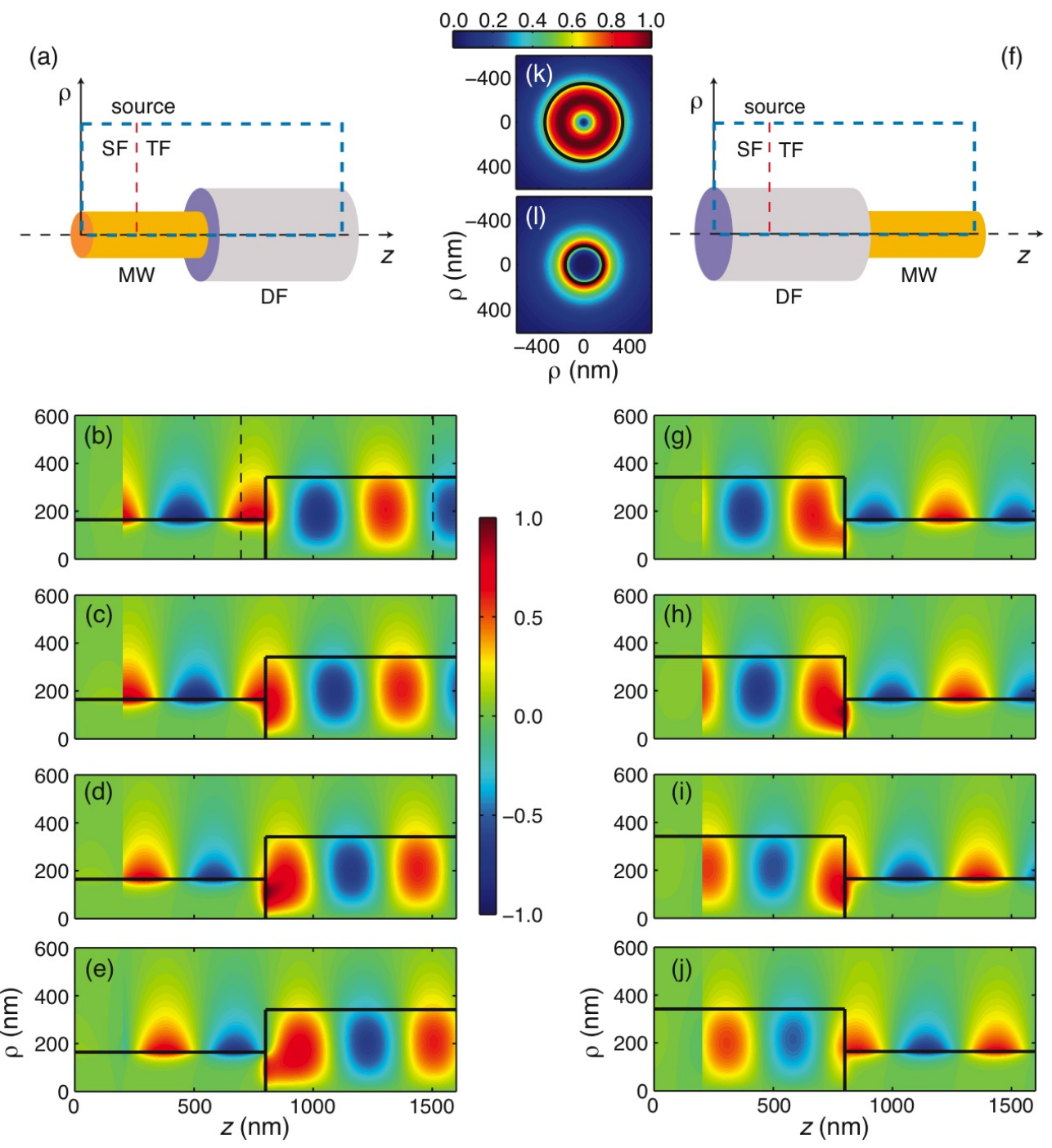

FIG. 1: (a) and (f) Layout of the coupling geometry. The vertical dashed red line indicates the separation between the total-field (TF) and the scattered-field (SF) region, and the position of the source. The area delimited by dashed lines is used in the FDTD simulations. (b)-(e) Snapshots of the magnetic field at times $t_{0}, t_{0}+\tau, t_{0}+2 \tau$, and $t_{0}+3 \tau$ respectively, for $\tau=0.25$ fs. The vertical dashed lines in (b) mark the area shown in 2(a). (g)-(j), Snapshots for coupling from the opposite direction. (k) and (l), Magnetic field patterns of the modes in the DF and MW, respectively. The radii of the silica DF and silver MW are $342 \mathrm{~nm}$ and $164 \mathrm{~nm}$, respectively, and the wavelength was set at $\lambda=633 \mathrm{~nm}$. 


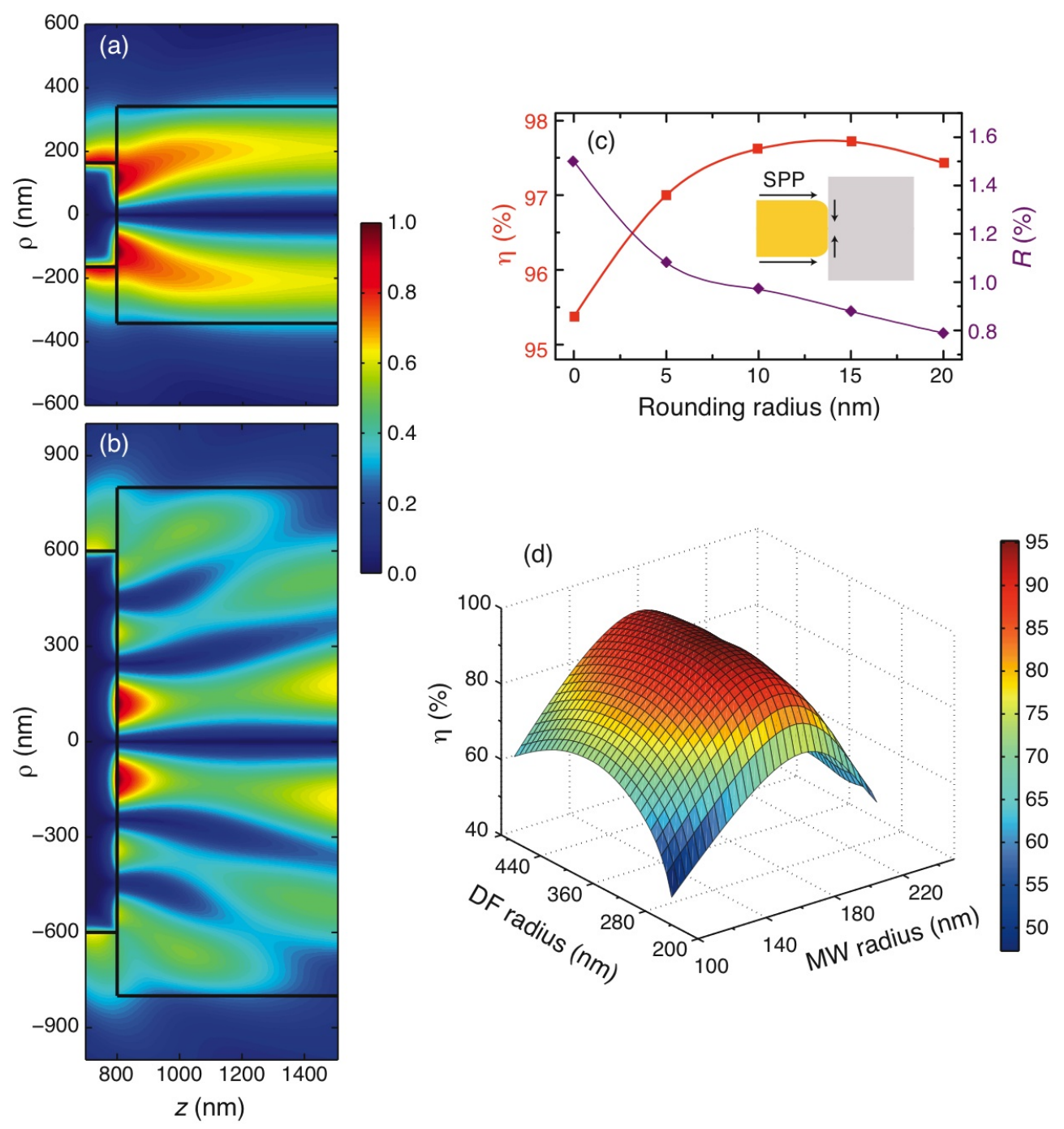

FIG. 2: (a) and (b) Time-averaged magnetic field of a silver MW coupled to a silica DF for two different sets of radii. See text for details. (c) The conversion efficiency $\eta$ and reflectivity $R$ as a function of the rounding radius of the silver MW for the parameters of 1 . The inset illustrates the geometry and the bending of SPPs onto the interface. (d) $\eta$ as a function of the MW and DF radii. The wavelength was set to $\lambda=633 \mathrm{~nm}$. 

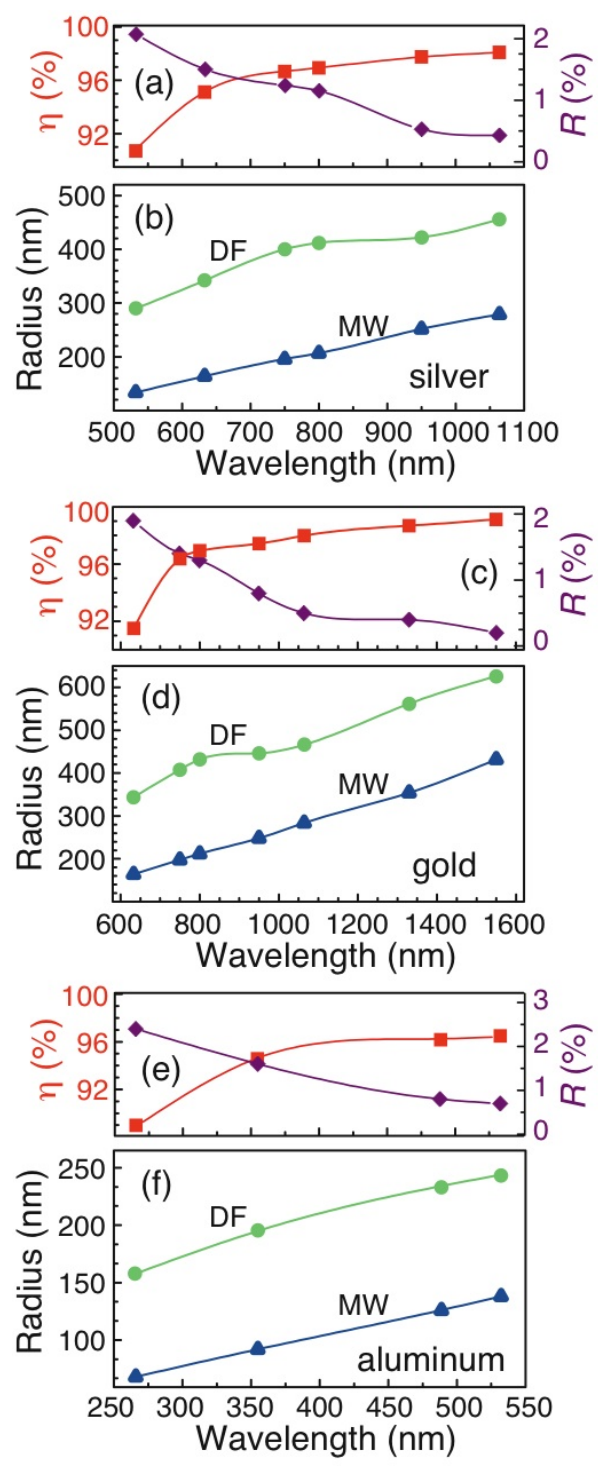

FIG. 3: Coupling efficiency and optimal radii of the MW and DF as a function of wavelength. (a) and (b) silver MW, (c) and (d) gold MW, (e) and (f) aluminium MW. 

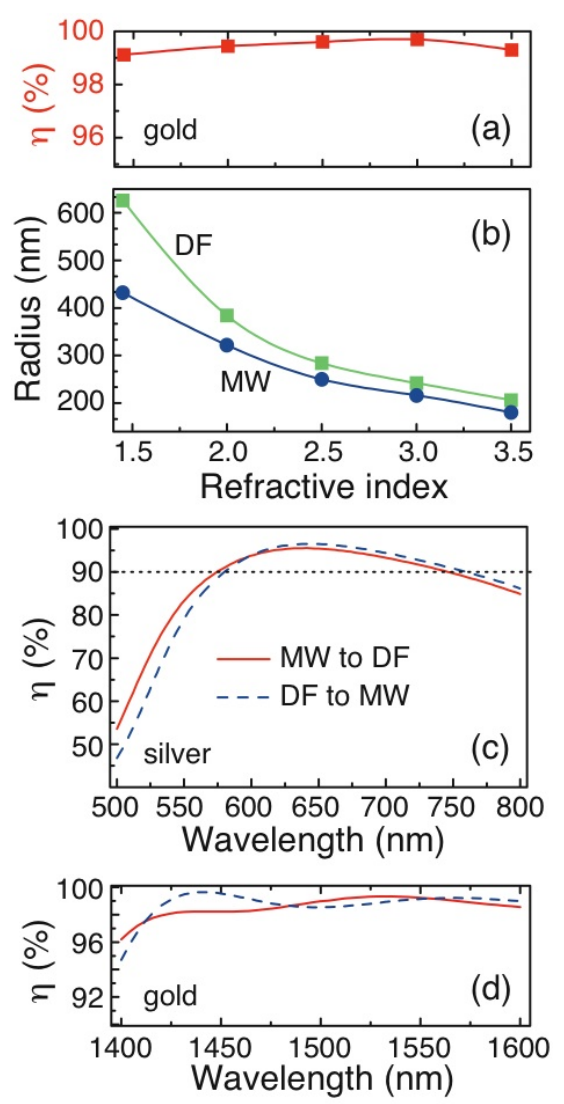

FIG. 4: (a-b) $\eta$ and optimal radii of a gold MW and DF as a function of the DF refractive index at $\lambda=1550 \mathrm{~nm}$. (c) $\eta$ for a silver MW of radius $164 \mathrm{~nm}$, coupled to a silica DF of radius $342 \mathrm{~nm}$ at $\lambda=633 \mathrm{~nm}$. (d) $\eta$ for a gold MW of radius $180 \mathrm{~nm}$ coupled to a silicon DF of radius $208 \mathrm{~nm}$ at $\lambda=1550 \mathrm{~nm}$. 

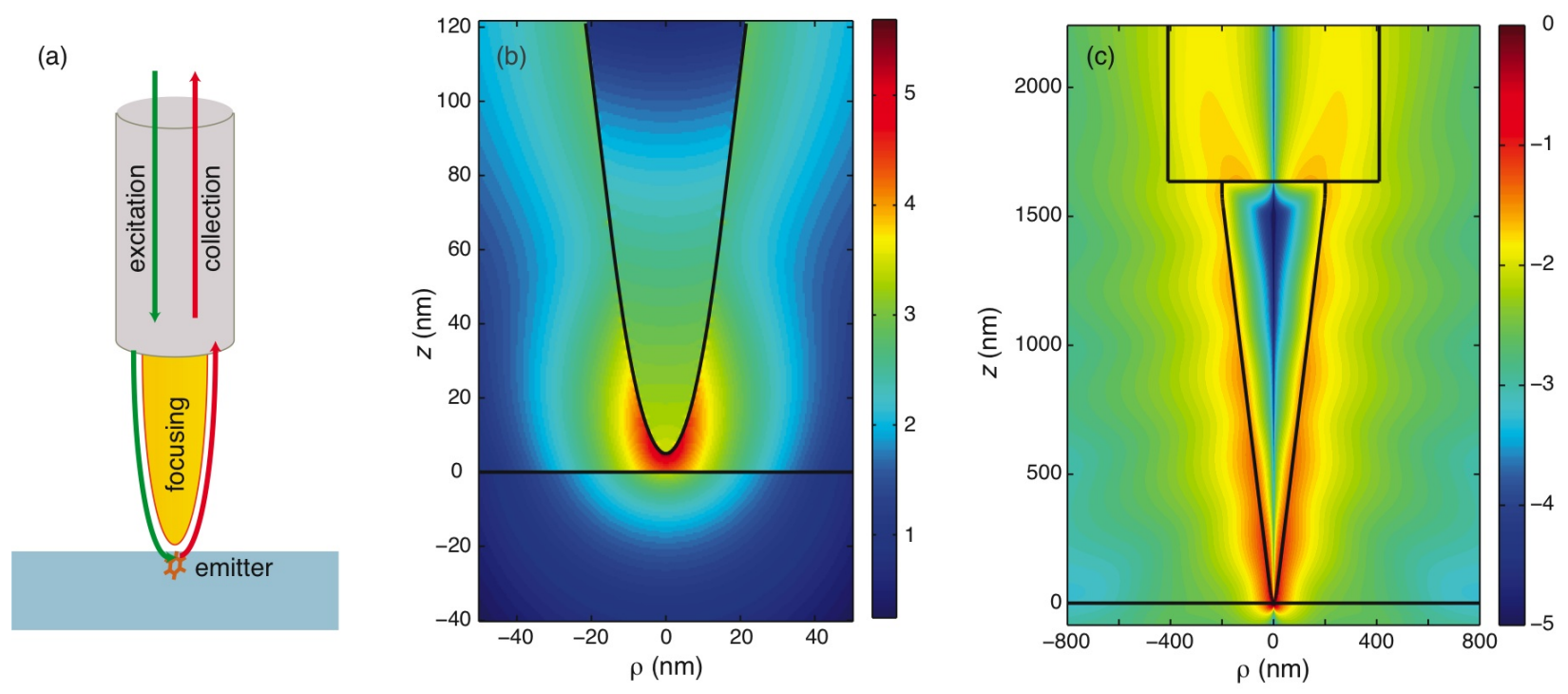

FIG. 5: (a) Sketch of the device consisting of a metallic nanocone butt-coupled to a DF. (b) Normalized distribution (logarithmic scale) of the electric field intensity near a substrate when photons are launched from the DF into the MW cone. (c) Time-averaged magnetic field radiated by a single emitter embedded in the substrate. See text for details. 